\title{
Towards the Independent Spanning Trees in Conditional BC Networks
}

\author{
Baolei Cheng ${ }^{1,2}$, Jianxi Fan ${ }^{1}$, Shukui Zhang ${ }^{1}$, Yan Wang ${ }^{1,2}$, Xi Wang ${ }^{1}$ and Wenjun Liu ${ }^{1}$ \\ ${ }^{1}$ School of Computer Science and Technology, Soochow University, Suzhou, 215006, China \\ ${ }^{2}$ Provincial Key Laboratory for Computer Information Processing Technology, Soochow University, Suzhou, 215006, China \\ \{chengbaolei, jxfan, zhangsk, wangyanme, 20124027002, 20114027003\}@ suda.edu.cn
}

\begin{abstract}
Independent spanning trees (ISTs for short) in networks have applications such as reliable communication protocols, the multi-node broadcasting, one-to-all broadcasting, reliable broadcasting, and secure message distribution. However, it is an open problem whether there are $n$ ISTs rooted at any node in any $n$-connected network with $n \geq 5$. In this paper, we consider the construction of ISTs in a family of hypercube variants, called conditional BC networks. A recursive algorithm based on two common rules is proposed to construct $n$ ISTs rooted at any node in any $n$-dimensional conditional BC network $X_{n}$. We also show that our constructive method is adaptive to not only the existing hypercube variants, but also some other ones.

Index Terms - Conditional BC network, independent spanning trees, node-disjoint paths, recursive algorithm.
\end{abstract}

\section{I . Introduction}

Interconnection networks play an important part in the parallel computing systems and an interconnection network can be denoted by a graph $G$ with the node set $V(G)$ and the edge set $E(G)$ [1-5]. A path $Q$ from $u$ to $v$ is also denoted as $\langle u, v\rangle$-path. We use $V(Q)$ and $E(Q)$ to denote the node set and the edge set in $Q$, respectively. Two spanning trees $T_{1}$ and $T_{2}$ rooted at a node $u$ in $G$ are independent if for each node $v \in V(G) \backslash\{u\}$, the $\langle u, v\rangle$-path $P$ in $T_{1}$ and the $<u, v>$-path $Q$ in $T_{2}$, then $V(P) \cap V(Q)=\{u, v\}$ and $E(P) \cap E(Q)=\phi$. A set of spanning trees in $G$ are independent if they are pairwisely independent. We use ISTs to denote independent spanning trees.

Recently, the problems to construct multiple ISTs in given graphs have received a lot of attention. Researchers have proved that ISTs has many applications such as reliable communication protocols [1], the multi-node broadcasting [5], one-to-all broadcasting [6], reliable broadcasting, and secure message distribution [7]. Nevertheless, there exists a key problem on ISTs in networks:

If a network is $n$-connected for $n \geq 1$, whether are there $n$ ISTs rooted at any node in this network [8]?

It was proved to be true [8-10] for $n \leq 4$, while it remains open for $n \geq 5$. Thus, researchers studied the existence of ISTs in special networks. This problem has been solved for some restricted classes of networks, such as product networks [11], hypercubes [12], [13], and some hypercube variants such as locally twisted cubes [14], crossed cubes [15], [16], Möbius cubes [17], [18], HCH cubes [19], twisted cubes [20].

However, there has so far been no work reported on a general method to construct ISTs in hypercube variants. In this paper, we study the construction of $n$ ISTs rooted at any node in $\mathrm{BC}$ networks with a condition, called conditional BC networks. Our major contributions in this paper are as follows:

1. We give the definition of $n$-dimensional condition $\mathrm{BC}$ network $X_{n}$ and discussed the domain of $X_{n}$.

2. We develop an algorithm to construct $n$ ISTs $T_{1}, T_{2}, \ldots$, and $T_{n}$ rooted at any node $u$ in $X_{n}$ for $n \geq 2$.

3. We prove that our algorithm adapts to not only the existing hypercube variants such as $Q_{n}, L T Q_{n}, T Q_{n}, C Q_{n}, M Q_{n}$, $\mathrm{HCH}_{n}$, but also some other ones such as $\mathrm{HMH}_{n}$.

\section{II . Preliminaries}

\section{A. Graph Terminologies}

Given a simple graph $G$ and a nonempty subset $V^{\prime} \subseteq V(G)$, we use $G\left[V^{\prime}\right]$ to denote the subgraph of $G$ induced by $V^{\prime}$. The address of each node in graph $G$ is represented by a unique binary string. Nodes and their addresses in $G$ will be used alternatively in this paper. A binary string $s$ with length $n$ will be written as $s_{n-1} s_{n-2} \ldots s_{0}$, where $s_{n-1}$ is the most significant bit and $s_{0}$ is the least significant bit.

\section{B. Definitions and Basic Lemmas}

Now we adopt the definition of bijective connection (BC for short) networks with addresses in their nodes from [21].

Definition 1. The 1-dimensional BC network $X_{1}$ is a complete network with two nodes 0 and 1 . The family of the 1dimensional BC network is defined as $L_{1}=\left\{X_{1}\right\}$. A network $G$ is an $n$-dimensional BC network, denoted by $X_{n}$, if there exist $V_{0}, V_{1} \subset V(G)$ such that the following conditions hold:

(1) $V_{0}=0 V_{0}^{\prime}$ and $V_{1}=1 V_{1}^{\prime}$, where $V_{0}^{\prime}=V_{1}^{\prime}=\{0,1\}^{n-1}$

\footnotetext{
* This work is supported by National Natural Science Foundation of China (61170021), Specialized Research Fund for the Doctoral Program of Higher Education (20103201110018), Natural Science Foundation of Jiangsu Province (BK2011376), Application Foundation Research of Suzhou of China (SYG201240,SYG201118), Scientific Innovation research of College Graduate in Jiangsu Province (CXZZ11_0100, No. CXLX12_0811), and sponsored by the Qing Lan Project.
} 
(2) $V(G)=V_{0} \cup V_{1}, V_{0} \neq \phi, V_{1} \neq \phi$, and $V_{0} \cap V_{1}=\phi$, and

(3) There exists a bijective connection between $V_{0}$ and $V_{1}$, $G\left[V_{0}\right] \in L_{n-1}$, and $G\left[V_{1}\right] \in L_{n-1}$.

The family of the $n$-dimensional BC networks is defined as $L_{n}=\{G \mid G$ is an $n$-dimensional BC network $\}$. Fig.1 shows two types of 3-dimensional BC network $X_{3}$.
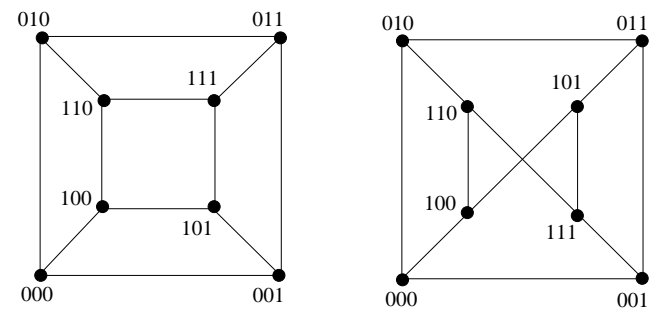

Fig. 1 (a) and (b) are two types of $X_{3}$.

If $u=u_{n-1} u_{n-2} \ldots u_{0}$ and $v=u_{n-1} u_{n-2} \ldots u_{d+1} \overline{u_{d}} v_{d-1} v_{d-2} \ldots v_{0}$ in a BC network $X_{n}$ are two adjacent nodes, then we say that $v$ is the $d$-neighbor of $u$. For this purpose, let $N(u, d)$ denote the $d$-neighbor of $u$ in $X_{n}$ for any integer $d$ with $0 \leq d \leq n-1$. Furthermore, if $W \subseteq V\left(X_{n}\right), N(W, d)=$ $\left\{u \in V\left(X_{n} \mid\right.\right.$ There exists $v \in W$, such that $\left.u=N(w, d)\right\}$. That is, $N(w, d)$ is a set of the $d$-neighbors of all the nodes in the set $W$. We define parent $(v, T)$ as the parent of node $v$ in a tree $T$. In order to facilitate this discussion, we can also use decimal number to denote the nodes in $X_{n}$.

We also adopt the definition of dimension-backbone tree from [18] to construct isomorphic trees.

Definition 2. Suppose that $T$ is a spanning tree rooted at node $u$ in $X_{n}$. Copy $T$ to $T^{\prime}$. For each node $y \in V(T) \backslash\{u\}$, if parent $(y, T)=N(y, m)$, we replace $y$ in $T^{\prime}$ with $m$. Then, the tree $T^{\prime}$ is called the dimension-backbone tree of $T$.

We give the definitions of ENS and ENSP as follows.

Definition 3. For any integer $n \geq 2$, suppose that $T_{1}$ is a spanning tree in $X_{n-1}^{u_{n-1}}$ rooted at node $u$ and graph $T_{2}$ is a spanning tree rooted at node $N(u, n-1)$ in $X_{n-1}^{\overline{u_{n-1}}}$. If there exists a nonempty set $W \subseteq V\left(T_{1}\right) \backslash\{u\}$ such that

(1) if $v \in W$, then ancestor $\left(v, T_{1}\right) \backslash\{u\} \subset W$, and

(2) $T_{2}[\{N(u, n-1)\} \bigcup N(W, n-1)]$ is a tree rooted at $N(u, n-1)$,

then we call $W$ an extendable node set with the abbreviation ENS with respect to the spanning tree $T_{1}$.

Definition 4. For any integer $n \geq 2$, let $T_{A, 1}, T_{A, 2}, \ldots, T_{A, n-1}$ be $n-1$ ISTs rooted at node $u$ in $X_{n-1}^{u_{n-1}}$ and $T_{B, 1}, T_{B, 2}, \ldots$, $T_{B, n-1}$ be $n-1$ ISTs rooted at $N(u, n-1)$ in $X_{n-1}^{\overline{u_{n-1}}}$. If the following conditions hold:

(1) $W_{1}, W_{2}, \ldots$, and $W_{n-1}$ are ENSes with respect to $T_{A, 1}$, $T_{A, 2}, \ldots, T_{A, n-1}$, respectively,

(2) For any two integers $i, j$ with $1 \leq i<j \leq n-1$, $W_{i} \cap W_{j}=\phi$, and

(3) $\bigcup_{i=1}^{n-1} W_{i} \bigcup\{u\}=V\left(X_{n-1}^{u_{n-1}}\right)$,

then we call $\left\{\{u\}, W_{1}, W_{2}, \ldots, W_{n-1}\right\}$ an extendable node set partition of $V\left(X_{n-1}^{u_{n-1}}\right)$ with the abbreviation ENSP.

\section{Algorithms to construct ISTs in conditional BC networks}

In this section, firstly, we give the definition of conditional $\mathrm{BC}$ networks by providing two common rules. Then, we design an alogorithm to construct independent spanning trees in the conditional BC netwoks.

For any $n \geq 2$, given a spanning tree $T_{1}$ rooted at $u$ in $X_{n-1}^{u_{n-1}}$, we will provide a rule to construct a graph $T_{1}{ }^{\prime}$, which is actually called by Rule 2 .

Rule 1. Suppose that $T_{1}$ is a spanning tree rooted at node $u$ in $X_{n-1}^{u_{n-1}} \cdot T_{1}^{\prime}$ is constructed by the following steps.

Step 1: Let $N(u, n-1)$ be the root of $T_{1}{ }^{\prime}$.

Step 2: Construct $T_{1}{ }^{\prime}$ such that $T_{1}{ }^{\prime}$ has the same dimensionbackbone tree with $T_{1}$.

Based on Rule 1, we will give Rule 2 to transform the problem of constructing ISTs in $X_{n}$ into the problems of constructing ISTs in $X_{n-1}^{u_{n-1}}$ and $X_{n-1}^{\overline{n_{n-1}}}$. To note that Rule 2 is a sub-procedure called by Algorithm XIST.

Rule 2. Suppose that $T_{A, 1}, T_{A, 2}, \ldots, T_{A, n-1}$ are $n-1$ ISTs rooted at node $u$ in $X_{n-1}^{u_{n-1}}$ and $T_{B, i}$ in $X_{n-1}^{\overline{u_{n-1}}}$ are obtained by using Rule 1 based on $T_{A, i}$ for $i=1$ to $n-1$. If the following conditions hold:

(1) $T_{B, 1}, T_{B, 2}, \ldots, T_{B, n-1}$ are $n-1$ ISTs rooted at $N(u, n-1)$ in $X_{n-1}^{\overline{u_{n-1}}}$.

(2) There exists an ENSP $\left\{\{u\}, W_{1}, W_{2}, \ldots, W_{n-1}\right\}$ on $V\left(X_{n-1}^{\overline{u_{n-1}}}\right)$, Then, $n$ ISTs $T_{1}, T_{2}, \ldots, T_{n}$ can be constructed by the following steps.

Step 1: for $i=1$ to $n-1$ do

$$
\begin{aligned}
& V\left(T_{i}\right)=V\left(T_{A, i}\right) \cup V\left(T_{B, i}\right) . \\
& E\left(T_{i}\right)=E\left(T_{A, i}\right) \bigcup E\left(T_{B, i}\right) .
\end{aligned}
$$




$$
\begin{aligned}
& E\left(T_{i}\right)=E\left(T_{A, i}\right) \backslash E\left(T_{B, i}\left[N\left(W_{i}, n-1\right)\right]\right) . \\
& E\left(T_{i}\right)=E\left(T_{i}\right) \bigcup\left\{(w, N(w, n-1)) \mid w \in W_{i}\right\}
\end{aligned}
$$

end for

Step 2: $\quad T_{B, n}=\bigcup_{i=1}^{n-1} T_{B, i}\left[N\left(W_{i} \cup\{u\}, n-1\right)\right]$.

$$
\begin{aligned}
& V\left(T_{n}\right)=V\left(T_{B, n}\right) \cup N\left(V\left(T_{B, n}\right), n-1\right) . \\
& E\left(T_{n}\right)=E\left(T_{B, n}\right) \bigcup\left\{(v, N(v, n-1)) \mid v \in V\left(T_{B, n}\right)\right\} .
\end{aligned}
$$

Lemma 1. Let $T_{A, 1}, T_{A, 2}, \ldots, T_{A, n-1}$ be $n-1$ ISTs rooted at node $u$ in $X_{n-1}^{u_{n-1}}$ and $T_{B, i}$ in $X_{n-1}^{\overline{u_{n-1}}}$ be obtained by using Rule 1 based on $T_{A, i}$ for $i=1$ to $n-1$. If $T_{B, 1}, T_{B, 2}, \ldots, T_{B, n-1}$ are $n-1$ ISTs rooted at $N(u, n-1)$ in $X_{n-1}^{\overline{u_{n-1}}}$ and $\left\{\{u\}, W_{1}, W_{2}, \ldots, W_{n-1}\right\}$ is an ENSP in $V\left(X_{n-1}^{\overline{u_{n-1}}}\right)$, then $T_{1}$, $T_{2}, \ldots, T_{n}$ constructed by Rule 2 are $n$ ISTs.

Definition 5. For any $\mathrm{BC}$ nework $X_{n}$, if $n$ independent spanning trees in $X_{n}$ can be constructed in recursive fashion by Rule 2 , then we call $X_{n}$ an $n$-dimensional conditional $B C$ network.

Now we provide a recursive algorithm to construct $n$ ISTs in conditional BC networks.

Algorithm XIST $\left(k, u_{n-1} u_{n-2} \ldots u_{0}\right)$

Input: An integer $k$ with $k \leq n$ and $u=u_{n-1} u_{n-2} \ldots u_{0}$ in a $n$ dimensional conditional BC network $X_{n}$, where $n \geq 2$.

Output: $k$ trees $T_{1}, T_{2}, \ldots, T_{k}$ rooted at node $u$ in $X_{n}$.

Begin

Step 1: if $k=1$ return a tree $T_{1}$ rooted at node $u$ with

$$
\begin{aligned}
& V\left(T_{1}\right)=\{u, N(u, 0)\} \text { and } E\left(T_{1}\right)=\{(u, N(u, 0))\} . \\
& \text { else XIST }\left(k-1, u_{n-1} u_{n-2} \ldots u_{0}\right) .
\end{aligned}
$$

Step 2: $T_{A, i}=T_{i}$ for $i=1$ to $k-1$.

Construct $T_{B, i}$ by Rule 1 based on $T_{A, i}$ for $i=1$ to $n-1$.

Step 3: Construct $T_{1}, T_{2}, \ldots, T_{k}$ rooted at node $u$ in $X_{n}$ by Rule 2.

end

By Lemma 1 and Algorithm XIST, we can verify the following theorem hold.

Theorem 1. Algorithm XIST constructs $n$ independent spanning trees rooted at any node $u$ in $n$-dimensional BC network $X_{n}$ with $n \geq 2$.

\section{IV . Domain of Conditional BC networks}

In [15], we present a recursive algorithm to construct
ISTs in crossed cubes, which is based on the transformation of the addresses between two adjacent nodes. Since any node $u$ and its $(n-1)$-neighbor $N(u, n-1)$ are similar nodes (that is, there is an automorphism that maps $N(u, n-1)$ to $u)$, we can easily verify that we can also use Algorithm XIST to construct ISTs rooted at $u$ in the $n$-dimensional crossed cube $C Q_{n}$. By [19] and [20], we can verify that Algorithm XIST can solve the IST problems in the $n$-dimensional $\mathrm{HCH}$ cube $\mathrm{HCH}_{n}$ and the $n$-dimensional twisted cube $T Q_{n}$, respectively. Similarly, we can verify Algorithm XIST can be applied to the $n$ dimensional hypercube $Q_{n}$ and the $n$-dimensional locally twisted cube $L T Q_{n}$. Thus, we have the following lemma.

Lemma 2. Let $\Gamma_{n}=\left\{X_{n} \mid X_{n}\right.$ is an $n$-dimensional conditional BC network \} for $n \geq 1$. Then,

$$
\Gamma_{n} \supseteq\left\{Q_{n}, L T Q_{n}, T Q_{n}, C Q_{n}, M Q_{n}, H C H_{n}\right\} .
$$

In the following, we will show that our constructive method is adaptive to not only the existing hypercube variants such as $L T Q_{n}, T Q_{n}, C Q_{n}, M Q_{n}, H C H_{n}$, but also some other ones. For this purpose, based on the definitions of hypercubes [12] and Möbius cubes [17], we propose $\mathrm{HMH}$ cubes as follows.

Definition 6. The $n$-dimensional HMH cube $H M H_{n}$ is $Q_{n}$ for any integer $n$ with $1 \leq n \leq 3$. For $n \geq 4, H M H_{n}$ is constructed by $Q_{n-1}$ and $0-M_{n-1}$ as follows.

(1) Let $Q_{n-1}^{1}$ denote the graph obtained by prefixing the value of each node of one copy of $Q_{n-1}$ with 1 and $M_{n-1}^{0}$ denote the graph obtained by prefixing the value of each node of the other copy of $0-M_{n-1}$ with 1 .

(2) For two nodes $x=1 x_{n-2} x_{n-3} \ldots x_{0} \in V\left(Q_{n-1}^{1}\right)$, $y=0 y_{n-2} y_{n-3} \ldots y_{0} \in V\left(M_{n-1}^{0}\right),(x, y) \in E\left(H M H_{n}\right)$ if and only if $x_{n-2} x_{n-3} \ldots x_{0}=y_{n-2} y_{n-3} \ldots y_{0}$.

In what follows, we use Algorithm XIST to construct ISTs in the network $H M H_{n}$. Fig. 2 shows $H M H_{4}$.

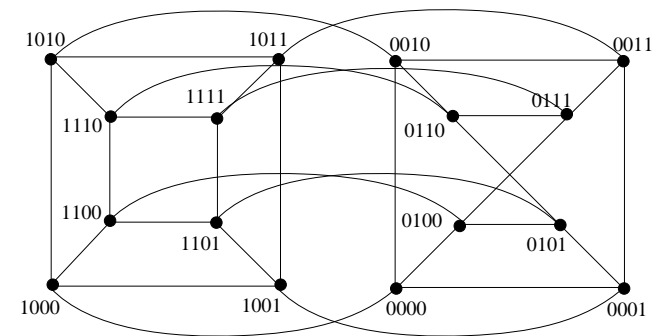

Fig. $2 \mathrm{HMH}_{4}$ 
If root $u$ is in $Q_{n-1}^{1}$, for $i=1$ to $n-1$, we let $W_{i}=\left\{1 u_{n-1} u_{n-2} \ldots \bar{u}_{i} x_{i-1} x_{i-2} \ldots x_{0} \in V\left(Q_{n-1}^{1}\right)\right\}$. Otherwise, we let $W_{i}=\left\{0 u_{n-1} u_{n-2} \ldots \bar{u}_{i} x_{i-1} x_{i-2} \ldots x_{0} \in V\left(M_{n-1}^{0}\right)\right\}$. It is easy to construct ISTs $T_{A, 1}, T_{A, 2}$, and $T_{A, 3}$ rooted at 1 in $M_{3}^{0}$. By Algorithm XIST, we can obtain $T_{B, 1}, T_{B, 2}$, and $T_{B, 3}$ rooted at node 9 in $Q_{3}^{1}$. Fig. 3(a) shows how to construct four ISTs $T_{1}$, $T_{2}, T_{3}$, and $T_{4}$ rooted at node 1 or 9 in $\mathrm{HMH}_{4}$ based on three ISTs $T_{A, 1}, T_{A, 2}$, and $T_{A, 3}$ rooted at node 1 in $M_{3}^{0}$, three ISTs $T_{B, 1}, T_{B, 2}$, and $T_{B, 3}$ rooted at node 9 in $Q_{3}^{1}$, and an ENSP $\{\{0\},\{2,3\},\{4,5,6,7\},\{1\}\}$ on $M_{3}^{0}$. Fig. 3(b) illustrates the forward direction example which shows the four ISTs rooted at 1 . Similarly, the four ISTs rooted at 9 with the backword direction are shown in Fig. 3(c).

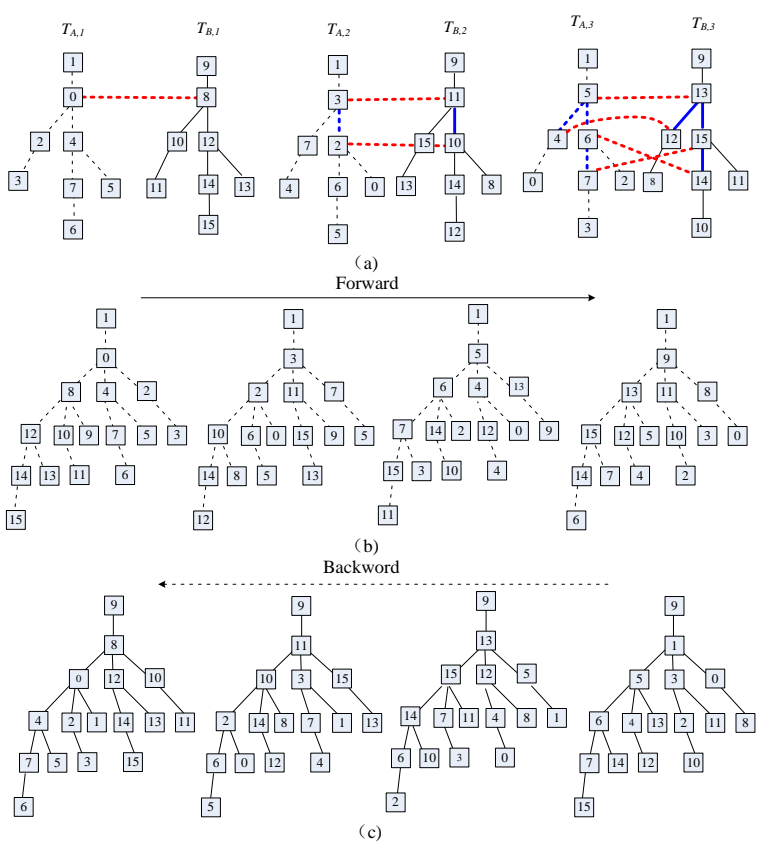

Fig. 3 Construction procedures of ISTs in $\mathrm{HMH}_{4}$.

Based Definition 5 and the above discussion, we have the following lemma.

Lemma 3. $H M H_{n} \in \Gamma_{n}$.

Based on Lemma 2, Lemma 3, and the above discussion, we have the following theorem.

Theorem 2. $\Gamma_{n} \supseteq\left\{H M H_{n}, Q_{n}, L T Q_{n}, T Q_{n}, C Q_{n}, M Q_{n}, \quad, H C H_{n}\right\}$.

\section{V . Conclusions}

In this paper, we have proposed a more general apporoch to construct $n$ ISTs in a family of of hypercube variants- conditional $\mathrm{BC}$ networks, which not only include the existing hypercube variants such as $Q_{n}, L T Q_{n}, T Q_{n}, C Q_{n}, M Q_{n}$, $\mathrm{HCH}_{n}$, but also include some other ones such as $H M H_{n}$.

\section{References}

[1] F. Bao, Y. Funyu, Y. Hamada, Y. Igarashi, "Reliable broadcasting and secure distributing in channel networks", IEICE T. Fund. Electr., vol. E81-A , pp. 796-806 (1998).

[2] J. Fan, X. Jia, X. Lin, "Optimal Embeddings of Paths with Various Lengths in Twisted Cubes", IEEE Trans. Parallel Distrib. Syst., vol. 18, no. 4, pp. 511-521 (2007).

[3] J. Fan, X. Jia, "Embedding meshes into crossed cubes", Inform. Sciences, vol. 177 , no. 15 , pp. $3151-3160$ (2007).

[4] J. Fan, X. Lin, X. Jia, R. W. H. Lau, "Edge-pancyclicity of twisted cubes", Lecture Notes in Computer Science, vol. 3827, pp. 1090-1099 (2005).

[5] Y.-S. Chen, C.-Y. Chiang, C.-Y. Chen, "Multi-node broadcasting in allported 3-D wormhole-routed torus using an aggregation-thendistribution strategy", J. Syst. Architect., vol. 50, no. 9, pp. 575-589 (2004).

[6] Y.-C. Tseng, S.-Y. Wang, and C.-W. Ho, "Efficient broadcasting in wormhole-routed multicomputers: A network-partitioning approach", IEEE Trans. Parallel Distrib. Syst., vol. 10, no.1, pp. 44--61(1999).

[7] F. Bao, Y. Igarashi, S.R. Öring, "Reliable broadcasting in product networks", Discrete Appl. Math., vol. 83, no. 1-3, pp. 3-20 (1998).

[8] A. Zehavi, A. Itai, "Three tree-paths", J. Graph Theor., vol. 13, no. 2, pp. 175-188, 1989.

[9] J. Cheriyan, S.N. Maheshwari, "Finding nonseparating induced cycles and independent spanning trees in 3-connected graphs", J. Algorithm., vol. 9, no. 4, pp. 507-537 (1988).

[10] S. Curran, O. Lee, X. Yu, "Finding four independent trees", SIAM J. Comput., vol. 35, no. 5, pp. 1023-1058 (2006).

[11] K. Obokata, Y. Iwasaki, F. Bao, Y. Igarashi, "Independent spanning trees of product graphs and their construction", IEICE T. Fund. Electr., vol. E79-A, no. 11, pp. 1894-1903 (1996).

[12] S.-M. Tang, Y.-L. Wang, Y.-H. Leu, "Optimal independent spanning trees on hypercubes", J. Inf. Sci. and Eng., vol. 20, no. 1, pp. 143-155 (2004).

[13] J. Werapun, S. Intakosum, V. Boonjing, "An efficient parallel construction of optimal independent spanning trees on hypercubes", $J$. Parallel Distr. Com., vol. 72, no. 12, pp. 1713-1724, 2012.

[14] Y.-J. Liu, W.Y. Chou, J.K. Lan, C. Chen, "Constructing independent spanning trees for locally twisted cubes", Theor. Comput. Sci., vol. 412, no. 22, pp. 2237-2252 (2011).

[15] B. Cheng, J. Fan, X. Jia, S. Zhang, "Independent spanning trees in crossed cubes", Inform. Sciences, vol. 233, no.1, pp. 276-289, 2013.

[16] B. Cheng, J. Fan, X. Jia, and J. Wang, "Dimension-adjacent trees and parallel construction of independent spanning trees on crossed cubes", $J$. Parallel Distr. Com., http://dx.doi.org/10.1016/j.jpdc.2013.01.009, 2013

[17] B. Cheng, J. Fan, X. Jia, S. Zhang, B. Chen, "Constructive algorithm of independent spanning trees on Möbius cubes", The Computer Journal (2012) http://dx.doi.org/10.1093/comjnl/bxs123.

[18] B. Cheng, J. Fan, X. Jia, and J. Jia, "Parallel construction of independent spanning trees and an application in diagnosis on Möbius cubes", $J$. Supercomput., http://dx.doi.org/10.1007/s11227-013-0883-1, 2013.

[19] B. Cheng, J. Fan, J. Yang, Y. Wang, and S. Zhang, "Independent spanning trees on special BC networks", Applied Mechanics and Materials, vol. 263-266, no. 1, pp. 3301-3305, 2013.

[20] Y. Wang, J. Fan, G. Zhou, X. Jia, "Independent spanning trees on twisted cubes", J. Parallel Distr. Com., vol. 72, no. 1, pp. 58-69, 2012.

[21] J. Fan, X. Jia, X. Liu, S. Zhang, J. Yu, "Efficient unicast in bijective connection networks with the restricted faulty node set", Inform. Sciences, vol. 181, no. 11, pp. 2303-2315 (2011). 